\title{
Efficient heat conducting liquid metal/CNT pads with thermal interface materials
}

\author{
LIUYING ZHAO ${ }^{1,2, *}$, SHENG CHU ${ }^{1,2}$, XUECHEN CHEN $^{1,3}$ and GUANG CHU ${ }^{4}$ \\ ${ }^{1}$ State Key Laboratory of Optoelectronic Materials and Technologies, School of Material Science and Engineering, \\ Sun Yat-Sen University, Guangzhou 510275, People's Republic of China \\ ${ }^{2}$ School of Materials Science and Engineering, Sun Yat-Sen University, Guangzhou 510275, People's Republic of China \\ ${ }^{3}$ School of Electronics and Information Technology, Sun Yat-Sen University, Guangzhou 510275, \\ People's Republic of China \\ ${ }^{4}$ School of Metallurgy and Environment, Central South University, Changsha 410083, People's Republic of China \\ *Author for correspondence (15198872657@126.com)
}

MS received 2 January 2019; accepted 19 February 2019; published online 14 June 2019

\begin{abstract}
Ga-Based thermal interface material (TIM) pads/sheets with high thermal conductivity $(\kappa)$ are indispensable components in thermal management systems. Here, we present a feasible method to fabricate heat conduction pads, which are composed of carbon nanotubes embedded into a liquid metal (LM). This setup has resulted in a large increase of $\kappa$ reaching $\sim 14.2 \mathrm{~W} \mathrm{mK}^{-1}$, greater than that of most of the commercial thermal silicone pads $\left(\sim 5 \mathrm{~W} \mathrm{mK}^{-1}\right)$. In addition, a series of experiments were conducted on smartphones to evaluate the heat dissipation performance of the CPU. It turned out that LM/nanotube pads with TIMs show distinguish thermal conductivity performance.
\end{abstract}

Keywords. Liquid metal; gallium oxide; carbon nanotubes; thermal conductivity; heat-dissipation.

\section{Introduction}

With the rapid development of electronic information technology, the integration level and assembly density of electronic components are constantly increasing. While providing powerful useful functions, the power consumption and heat generation of the electronic devices are drastically increasing [1-5]. High temperatures will definitely lead to a detrimental effect on the stability, reliability and longevity of electronic components. Generally, in the range of $70-80^{\circ} \mathrm{C}$, the reliability of electronic equipment is reduced by more than $10 \%$ for every $1{ }^{\circ} \mathrm{C}$ increase, which means the higher the temperature, the greater the degradation [6]. According to incomplete statistics, the failure rate of an electronic equipment caused by excessive temperature accounts for more than $80 \%$ [7]. Therefore, it is essentially crucial for microelectronic products to improve their heat dissipation capacity which was generated by a heat source and ensure that the heat is discharged in a timely manner [8,9]. For electronic devices, central processing unit (CPU) will produce a lot of heat when working. If this heat could not be sent out in time, it may bring about a dead machine and burn down the CPU. A radiator/heatsink is used to dissipate the heat from the CPU. So a radiator/heatsink plays a decisive role in the stable operation of a CPU. Under normal circumstances, concave, convex or wavy space is present between the surface of the microelectronic material and the radiator, as shown in figure 1a (red and black areas). So once they are installed together directly, the actual contact area between the heat source and the heat sink is only about $10-30 \%$ [10] and the rest is air gap.

The thermal conductivity of air is only $0.026 \mathrm{~W} \mathrm{mK}^{-1}$ [11], which could cause a very large thermal resistance between the electronic elements (CPU) and the radiator. As a consequence, the air gap seriously hinders the heat conduction and ultimately results in a low efficiency of the radiator $[12,13]$. Among the various dissipation heat forms, thermal interface materials (TIMs) play a key role in thermal management. TIMs are a kind of material for semiconductor packaging and electron heat dissipation. The common TIMs (silicon grease) are mainly composed of polymers and metal particle fillers, e.g., silver ( $\mathrm{Ag})$ [14], aluminium oxide $\left(\mathrm{Al}_{2} \mathrm{O}_{3}\right)[15,16]$, boron nitride (BN) $[17,18]$ and aluminium nitride (AlN) [19]. In fact, polymers do not conduct heat or the $\kappa$ is quite low. Moreover, there is too much thermal interface resistance between the particle/polymer interface (figure 1b) [20]. Even with a very high particle filler loading (up to 80 vol\%) such composites still cannot achieve a desirable $\kappa\left(<6 \mathrm{~W} \mathrm{mK}^{-1}\right)$ as shown in supplementary figure $S 1[21,22]$. In particular, a too high filling ratio will deteriorate the mechanical elasticity of TIMs. The diagram of ideal TIMs is shown in figure 1a. 
(a)

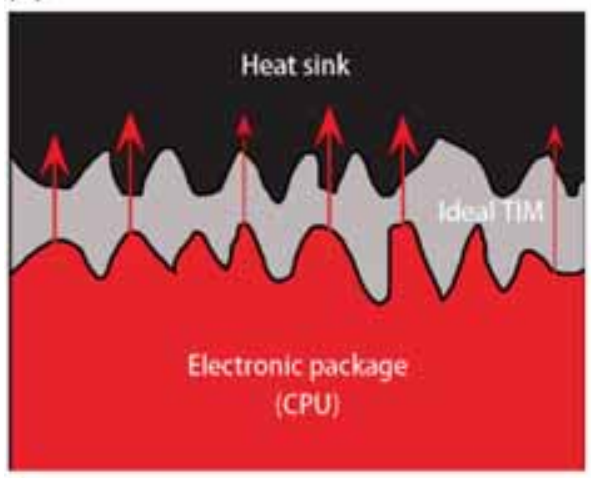

(c)

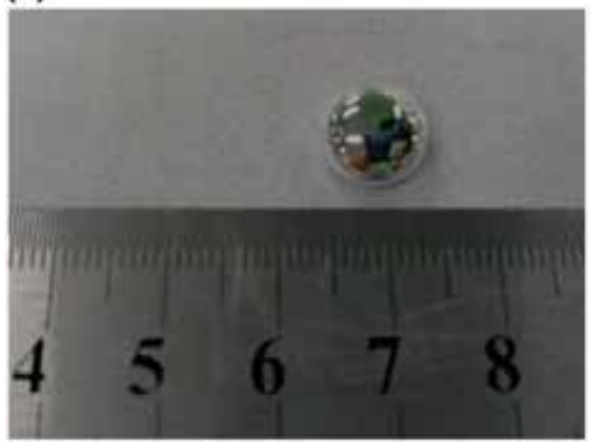

(b)

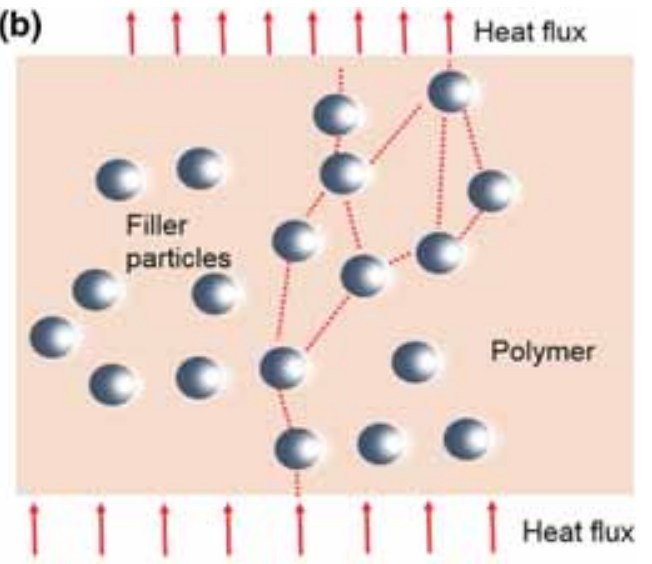

(d)

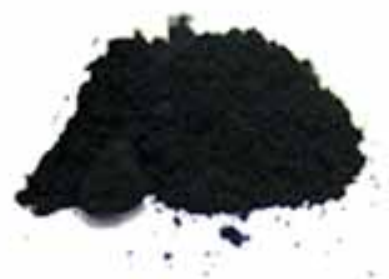

Figure 1. (a) Exploded view of the thermal interface when an ideal TIM is used. (b) Section drawing of common heat-conducting interface materials, where high $k$ particles are used as fillers. (c) Photograph of LM gallium. (d) Image of CNTs.

Recently, interest in liquid metals (LMs) such as eutectic GaIn (75 wt $\%$ gallium and $25 \mathrm{wt} \%$ indium) and galliumindium-tin (Galinstan, $68.5 \mathrm{wt} \%$ gallium, $21.5 \mathrm{wt} \%$ indium and $10 \mathrm{wt} \%$ tin) has grown continuously [23-27]. A LM is an amorphous, fluidity of the metal (figure 1c). The thermal conductivity of a LM is relatively higher than that of a traditional thermal conductive polymer. In addition, a LM does not contain volatile matter or toxic gas (such as silicon oil), so it is safe, stable and reliable and can guarantee the long-term safe and stable operation of the heat dissipation system. So far, LMs have been showing great application prospects [28-31]. Progress has been made in the study of LMs as heat conducting interface materials. LM/PDMS pads demonstrated unique advantages, which resulted in an outstanding $\kappa\left(8.3 \mathrm{~W} \mathrm{mK}^{-1}\right)$ by Zhao et al [32]. Furthermore, LM/Ag pads showed a thermal conductivity of $46 \mathrm{~W} \mathrm{mK}^{-1}$ by Lin et al [14]. These achievements indicate that LMs play a more important role in the field of heat conduction.

Since the discovery of carbon nanotubes (CNTs) in 1991 by Iijima [33], on account of their excellent mechanical, electrical and optical properties, CNTs (figure 1d) have attracted more and more attention [34-36]. Besides, CNTs possess remarkable thermal conductivity which makes them a good additive for heat conduction materials or heat conduction composite materials [37-39]. The thermal conductivity of CNTs is mostly based on the theoretical simulations and calculations from indirect experiments, providing a significant result: the $\kappa$ usually is in the range of $2000-6000 \mathrm{~W} \mathrm{mK}^{-1}$ [37-40]. Moreover, CNTs are one of the most ideal functional fillers for heat conduction and heat dissipation. Therefore, a composite with LM/CNTs is expected to be an excellent heat conduction material [41-43].

In this paper, solid metal gallium was melt and stirred in air for $36 \mathrm{~h}$ to obtain a gel-like state gallium oxide, which could reduce the fluidity and increase the wettability of the LM. In order to improve the thermal conductivity, CNTs are incorporated into the gel-like state gallium oxide. For solving the leakage problem of the $\mathrm{LM}$, a heat conducting rubber frame is adopted to bind with the LM. The LM/CNT composite was prepared as a thermal pad and tested. Finally, the pad was installed into a smartphone to evaluate its heat conduction performance.

\section{Experimental}

\subsection{Gel-like state gallium oxide preparation}

Gallium ingot (99.999\%, Wuhan xinrong new material co. Ltd) was heated for $1 \mathrm{~h}$ at $80^{\circ} \mathrm{C}$ by using a muffle furnace (MFLGM308-17, Hangzhou sanyongde) and then the LM was transferred to a plastic bottle and stirred for $36 \mathrm{~h}$ 
(a)

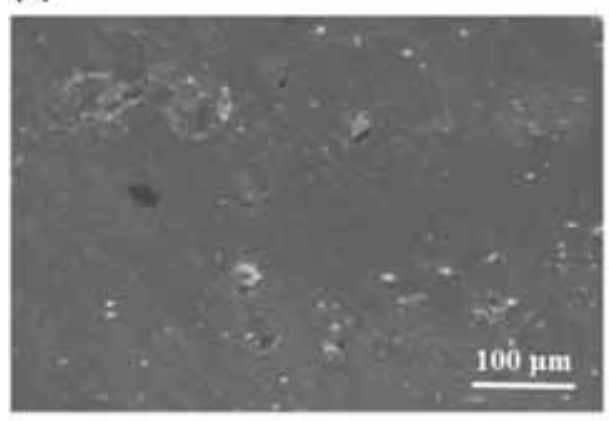

(c)

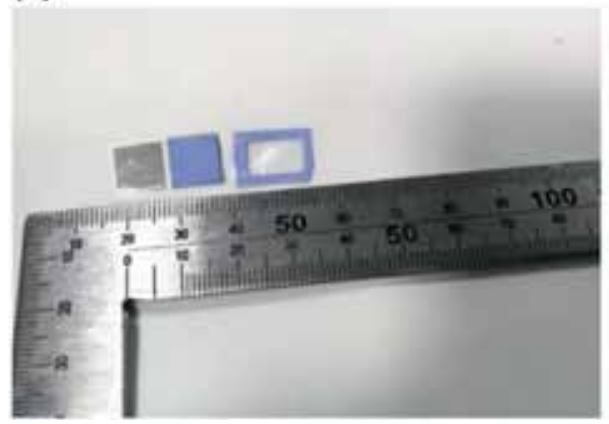

(b)

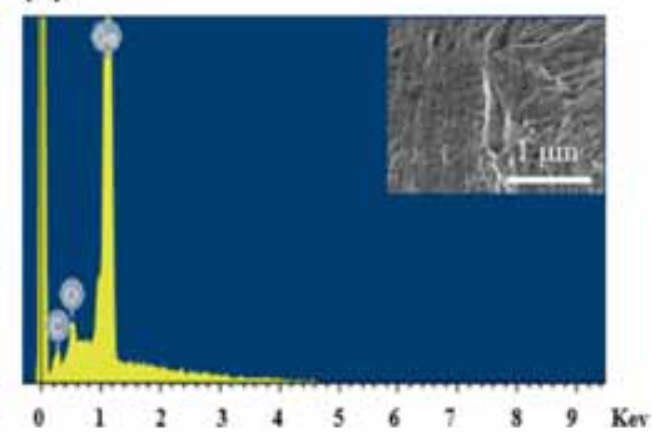

(d)

Figure 2. (a) SEM image of the LM/CNT composites. (b) EDS of the LM/CNTs, scale bar, $1 \mu \mathrm{m}$. (c) LM/CNT pad (left), silicone pad (middle) and silicone frame (right). (d) Schematic diagram of spill-resistant protecting rubber frame and LM/CNT thermal pad.

Table 1. Relationship between the mass ratio of CNTs and their thermal conductivity and thermal resistance.

\begin{tabular}{lllllc}
\hline Mass percentage $(\%)$ & 0 & 0.00625 & 0.0125 & 0.025 & 0.05 \\
Thermal resistance $\left(10^{-4} \mathrm{~m}^{2} \mathrm{~K} \mathrm{~W}^{-1}\right)$ & 6 & 1.3 & 1.0 & 0.35 & 0.38 \\
Thermal conductivity $\left(\mathrm{W} \mathrm{mK}^{-1}\right)$ & 2.0 & 3.8 & 5.0 & 14.2 & 13.3 \\
\hline
\end{tabular}

at $600 \mathrm{rpm}$ using a thermostatic stirrer (DF-II/DF-101S, Xinrui yiqi).

\subsection{LM/CNT composite preparation}

The CNTs (TF-2205, Tanfeng science and technology) were added into the gallium oxide according to different proportions $(0,0.00625,0.0125,0.025$ and $0.05 \%$, by weight $)$ and stirred for $24 \mathrm{~h}$ to ensure that all the CNTs are dispersed into the LM.

\section{Results and discussion}

At room temperature and pressure, the melting point of gallium is $29.7^{\circ} \mathrm{C}$. When touched by hand directly, the gallium ingot would melt from the solid state to liquid state. After melting completely, gallium presents a good fluidity and bright metallic luster of silver (figure 1c). Figure 2a shows the scanning electron microscopy (SEM) image of LM/CNTs, which is shown in black and gray. Figure $2 \mathrm{~b}$ shows the corresponding energy-dispersive X-ray spectroscopy (EDS) pattern of the LM/CNT composite, showing $\mathrm{Ga}, \mathrm{C}$ and $\mathrm{O}$ peaks. The EDS mapping images of $\mathrm{Ga}, \mathrm{C}$ and $\mathrm{O}$ elements (supplementary figure S2) further verify that CNTs are indeed dispersed into the LM. On the other hand, one could clearly see that CNTs have been doped with a LM from the inset of figure $2 b$.

The mixture of the CNTs and gel-liquid gallium oxide was transferred into a mould, then laminated and cut into a defined dimension shape for the smartphone. Figure $2 \mathrm{c}$ (left) shows the LM/CNT pad and figure 2c (middle) shows a common commercial thermal silicon pad (T-flex 600, $\kappa=$ $3.0 \mathrm{~W} \mathrm{mK}^{-1}$ ). Figure $2 \mathrm{c}$ (right) shows a thermal paste frame which is made from T-flex 600 .

The thermal resistance of the sample was tested by using a thermal resistance measuring instrument (DRL-III, Xiangtan xiangyi). The $\kappa$ of the sample with different contents of CNTs (in order to observe the experimental phenomena, we doubled the mass of CNTs for each experiment) is 


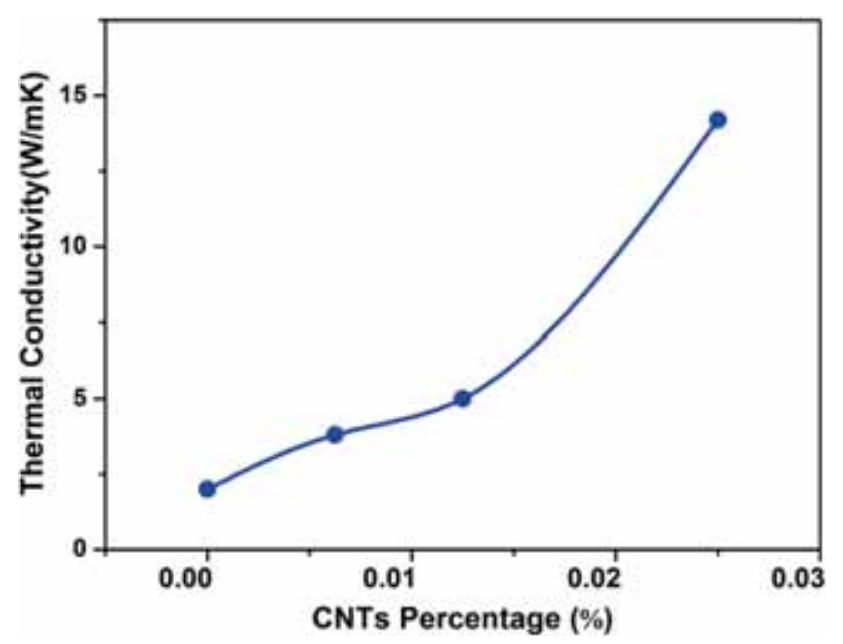

Figure 3. The $\kappa$ of the composites with different quantities of CNT contents.

summarized in table 1. It can be clearly seen that with the mass of the CNTs increasing, the thermal conductivity of the samples increases: the thermal conductivity of the sample at $0.0 \%$ is $2 \mathrm{~W} \mathrm{mK}^{-1}$, and when the mass ratio of CNTs is $0.025 \%$, the thermal conductivity of the sample reaches the maximum $\left(\kappa=14.2 \mathrm{~W} \mathrm{mK}^{-1}\right)$. Then, with a continuous increase $(0.05 \%)$ of the mass ratio of CNTs, there is no increase in thermal conductivity of the sample, which means that the LM has reached saturation. The relationship between the mass ratio of the CNTs and the thermal conductivity is shown in figure 3.

To verify the serviceability of TIMs for devices, a LM/CNT pad $(14 \mathrm{~mm} \times 12 \mathrm{~mm} \times 500 \mu \mathrm{m})$ was installed in the smartphone, as shown in figure $4 \mathrm{a}$. Then the smartphone was placed in an environment of constant temperature $\left(25^{\circ} \mathrm{C}\right)$ and relative humidity (45\%). During the test, the StabilityTest v 2.7 was run on the smartphone to fully operate its CPU. In order to prevent the leakage of the LM, the pad was surrounded by a conducting rubber frame (figure $2 \mathrm{~d}$ ). The configuration of a $\mathrm{LM} / \mathrm{CNT}$ thermal pad measurement unit for a smartphone is shown in figure $4 \mathrm{~b}$. As for the thermal management of the smartphone, the CPU temperature is the main monitoring data. Because CPU is the brain and heart of the smartphone, any temperature changes affect the performance of the smartphone directly. Figure $4 \mathrm{c}$ shows the CPU temperature rise when the heat load was applied and until the battery runs out. From figure $4 \mathrm{c}$ one can see that under the same thermal shock, the CPU with LM/CNT pads has an obvious smaller temperature rise, and the temperature drop reaches $2.8^{\circ} \mathrm{C}$ of (a)

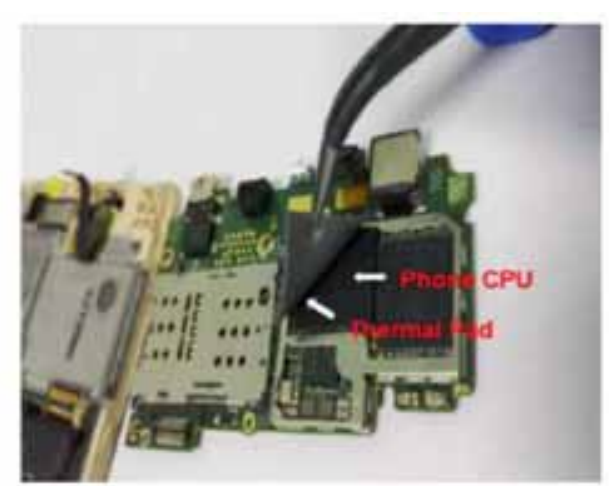

(c)

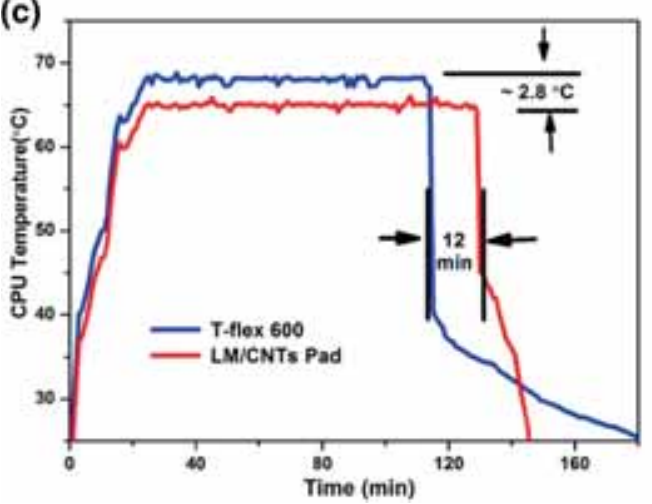

(b)

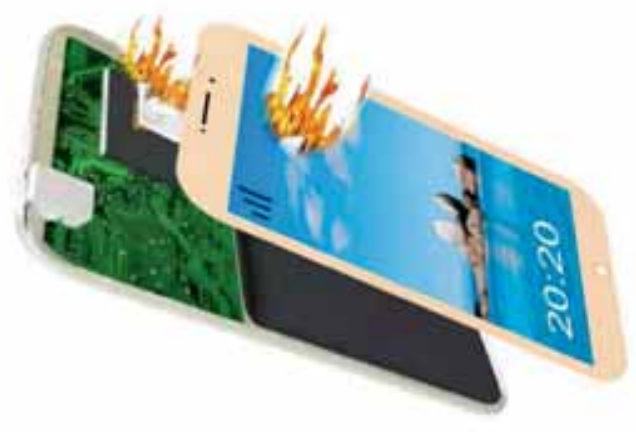

(d)

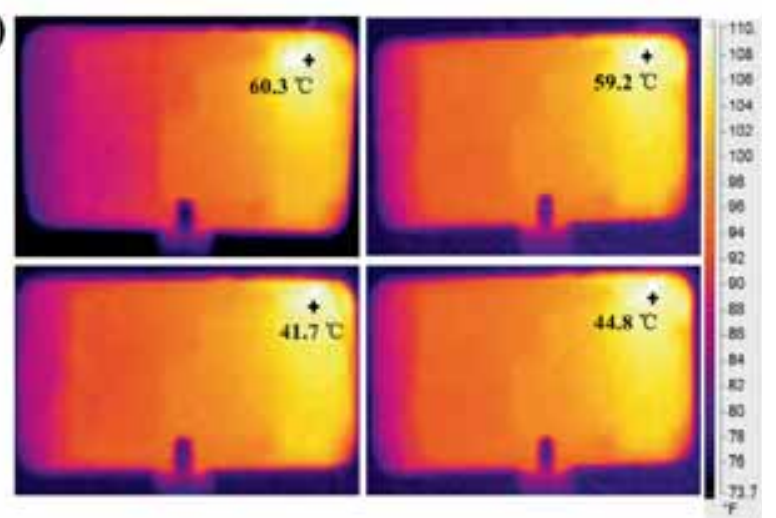

Figure 4. (a) Photograph image of the heat-dissipation measurement system. The LM/CNTs and T-flex 600 were cut into layers as thermal pads for application in a smartphone. (b) The demo of the thermal conductive pad with LM/CNTs as TIM of a smartphone. (c) Measured CPU temperature running time of smartphone with the T-flex 600 pad and LM/CNT pad, respectively. (d) Infrared thermal images of the front cover and back cover of the same smartphone under T-flex 600 pad (up) and LM/CNT pad (down) conditions. 
CPU, compared with a commercial T-flex 600 pad under the same conditions. Furthermore, the system with LM pads sustained 12 min longer than the silicone pad. This means that the LM/CNT pad has much better heat dissipation performance under instantaneous thermal shock since it has a smaller thermal resistance. At a steady running state, an infrared thermometer (Tis20, FLUKE) was used to detect the temperature map of the front cover and back cover of the smartphone. It is worth noting that while the back cover temperature is lowered down by $1.9^{\circ} \mathrm{C}$, the front screen temperature increased to about $1.1^{\circ} \mathrm{C}$, as shown in figure $4 \mathrm{~d}$. This is because much heat is transferred forward by the LM thermal pad since the overall amount of heat cannot be changed. However, the user fingers mainly come in contact with the back side of the phone and will generally not feel the temperature increase at the screen, and thus the user experience improves when using the LM/CNT pads.

\section{Conclusion}

In summary, heat dissipation TIMs were prepared from LM/CNTs. The composite pad demonstrates an outstanding $\kappa$ $\left(14.2 \mathrm{~W} \mathrm{mK}^{-1}\right)$. The heat dissipation performance of the pad was tested on a smartphone, which further proved that there is an obvious temperature decrease of $2.8^{\circ} \mathrm{C}$ at the CPU and $1.9^{\circ} \mathrm{C}$ at the back cover compared with a commercial thermal silicon pad. More importantly, the LM/CNT pad enhanced the battery running time by $12 \mathrm{~min}$, which indicates that this LM/CNT pad creates a meaningful impact on thermal management.

\section{Acknowledgements}

The authors acknowledge financial support from the National Science Foundation of China (Grant Nos. 11204097 and U1530120).

\section{References}

[1] David G C, Wayne K F, Kenneth E G, Gerald D M, Majumdar A, Humphrey J M et al 2003 J. Appl. Phys. 93793

[2] Prasher R 2006 Proc. IEEE 941571

[3] Andrew J M, Joshi Y K and Zhang Z M M 2012 Int. J. Therm. Sci. 622

[4] Ferain I, Colinge C A and Colinge J P 2011 Nature 479310

[5] Stassen I, Burtch N, Talin A, Falcaro P, Allendorf M and Ameloot R 2017 Chem. Soc. Rev. 463185

[6] Cohen A B, Kraus A D and Davidson S F 1983 Equip. Mech. Eng. 53150

[7] Kraus A D and Cohen A B 1983 Thermal analysis and control of electronic equipment (Washington, DC: Hemisphere Publishing Corp)

[8] Xu Y S, Luo X C and Chung D D L 2002 J. Electron. Packag. 124188
[9] Wolff E G and Schneider D A 1998 Int. J. Heat Mass Transfer 413469

[10] Gwinn J P and Webb R L 2003 J. Microelectron. 34215

[11] Yang D J, Zhang Q, Chen G, Yoon S F, Ahn J, Wang S G et al 2002 Phys. Rev. B 661

[12] Nieh H M, Teng T and Yu C C 2014 Int. J. Therm. Sci. 77252

[13] Alshaer W G, Nada S A, Rady M A, Barrio E P D and Sommier A 2015 Int J. Therm. Sci. 8979

[14] Lin Z Y, Liu H Q, Li Q G, Liu H, Chu S and Yang Y H 2018 Appl. Phys. A 1241

[15] Zhou W Y, Qi S H, Tu C C, Zhao H Z, Wang C F and Kou J L 2007 Appl. Polym. Sci. 1041312

[16] Hill R F and Supancic P H 2002 J. Am. Ceram. Soc. 85851

[17] Yung K C and Liem H 2007 J. Appl. Polym. Sci. 1063587

[18] Zhi C Y, Bando Y S, Terao T, Tang C C, Kuwahara H and Golberg D 2009 Adv. Funct. Mater. 191857

[19] Lee E S, Lee S M, Shanefield D J and Cannon W R 2008 J. Am. Ceram. Soc. 911169

[20] Wang Q, Gao W and Xie Z M 2003 J. Appl. Polym. Sci. 89 2397

[21] Leea G W, Parka M, Kima J, Leeb J I and Yoon H 2006 Compos. A: Appl. Sci. Manuf. 37727

[22] Yu W, Xie H Q, Yin L Q, Zhao J C, Xia L G and Chen L F 2015 Int. J. Therm. Sci. 9176

[23] Bartlett M D, Kazem N, Powell P M J, Huang X N, Sun W H, Malen J A M et al 2017 PANS 131

[24] Liang S Q, Li Y Y, Chen Y Z, Yang J B, Zhu T P, Zhu D Y et al 2017 J. Mater. Chem. C 515867

[25] Zhu S, So J H, Mays R, Desai S, Barnes W R, Pourdeyhimi B et al 2013 Adv. Funct. Mater. 232308

[26] Khan M R, Hayes G J, Zhang S L, Michael D D and Lazzi G 2012 IEEE Microw. Wirel. Compon. Lett. 22577

[27] Khoshmanesh K, Tang S Y, Zhu J Y, Schaefer S, Mitchell A and Kourosh K Z 2017 Lab Chip. 17974

[28] Larsson D H, Lundström U, Westermark U K, Henriksson M A, Burvall A and Hans M H 2013 Med. Phys. 40021909

[29] Yang X H and Liu J 2018 Front. Energy 12259

[30] Ge H S, Li H Y, Mei S F and Liu J 2013 Renew. Sustain. Energy Rev. 21331

[31] Wang J, Zhao X J, Cai Y X, Zhang C and Bao W W 2015 Energy Convers. Manage. 101532

[32] Zhao L Y, Liu H Q, Chen X C, Chu S, Liu H, Lin Z Y et al 2018 JMCC 610611

[33] Iijima S 1991 Nature 35456

[34] Wang Q and Arash B 2014 Comput. Mater. Sci. 82350

[35] Eatemadi A, Daraee H, Karimkhanloo H, Kouhi M, Zarghami N, Akbarzadeh A et al 2014 Nanoscale Res. Lett. 91

[36] Chen H Y, Ginzburg V V, Yang J, Yang Y F, Liu W, Huang Y et al 2016 Prog. Polym. Sci. $\mathbf{5 9} 41$

[37] Hong W T and Tai N H 2008 Diam. Relat. Mater. 171577

[38] Lin W, Moon K S and Wong C P 2009 Adv. Mater. 212421

[39] Gou Y J, Liu Z L, Zhang G M and Li Y X 2014 Int. J. Heat Mass Transfer $\mathbf{7 4} 358$

[40] Stroscio M A, Dutta M, Kahn D and Kim K W 2001 Superlattices Microstruct. 29405

[41] Grujicic M and Cao B G 2004 Mater. Sci. Eng. B 107204

[42] Hepplestone S P, Ciavarella A M, Janke C and Srivastava G P 2006 Surf. Sci. $\mathbf{6 0 0} 3633$

[43] Nan C W, Shi Z and Lin Y 2003 Chem. Phys. Lett. 375666 